\title{
Ovarian Carcinosarcoma with Yolk Sac Tumor in a Post-Menopausal Female: A Case Report and Review of Literature
}

\author{
${ }^{1}$ Institute of Pathology, St. Luke's Medical Center, Quezon City, Philippines \\ ${ }^{2}$ Cancer Institute, St. Luke's Medical Center, Quezon City, Philippines
}

Margarita Rae Rosario, ${ }^{1}$ Jeffrey So, ${ }^{1}$ Claire Anne Therese Hemedez, ${ }^{1}$ Carlos Dy ${ }^{2}$

\section{ABSTRACT}

Yolk sac tumor is the second most common subtype of ovarian germ cell tumors and is rare in postmenopausal women. The few cases in literature have found that in this age group, yolk sac tumors more commonly present as a mixed component, combined with epithelial tumors. We report a case of a 60 -yearold female who presented with an enlarging abdominopelvic mass. Imaging pointed to an ovarian new growth. Total abdominal hysterectomy with bilateral salpingo-oophorectomy revealed a tumor with three populations composed of carcinomatous, sarcomatous, and germ cell components, which was ultimately diagnosed as an ovarian carcinosarcoma with concurrent yolk sac tumor based on histomorphology and immunohistochemical staining. This report also discusses the proposed pathogenesis, treatment, and prognosis of this uncommon entity.

Key words: carcinosarcoma, yolk sac tumor, ovary, postmenopausal

ISSN 2507-8364 (Online)

Printed in the Philippines. Copyright $(2) 2021$ by the PJP.

Received: 15 October 2021

Accepted: 12 December 2021

Published online first: 17 December 2021.

https://doi.org/10.21141/PJP.2021.18

Corresponding author: Margarita Rae N. Rosario, $M D$ E-mail:marga.rae.rosario@gmail.com

ORCiD: https://orcid.org/0000-0001-6484-5123

\section{INTRODUCTION}

Ovarian yolk sac tumors (YSTs), formerly known as endodermal sinus tumors, are the third most common ovarian malignant germ cell tumors (GCTs), accounting for approximately $1 \%$ of ovarian malignancies and $14.5 \%-$ $16.4 \%$ of all ovarian malignant GCTs. ${ }^{1}$ They usually occur in the second to third decades of life and are rare in postmenopausal women. The majority of YSTs arising in old age tend to concurrently accompany variable types of somatic epithelial neoplasms. Rutgers et al. (1987) were the first to report a case of ovarian epithelial cancer associated with YST. ${ }^{2}$ It was only until Nogales et al. in 1996 when a YST was reported to occur with malignant Müllerian mixed tumor (MMMT). ${ }^{3}$ Since then, there have only been 5 reported cases in English literature in which YST was admixed with epithelial and spindle cell components in postmenopausal women (Table 1). We report a case of a 60-year-old post-menopausal female who presented with an enlarging abdominopelvic mass, ultimately diagnosed as a case of ovarian carcinosarcoma with concurrent YST based on histomorphology and immunohistochemical staining.

Table 1. Mixed yolk sac tumors with epithelial component in
post-menopausal women (adopted from Roth et al., 2011)

\section{CASE}

The case is a 60-year-old postmenopausal patient who presented with a two-month history of enlarging abdominal girth, especially at the right lower quadrant. Physical 
examination upon admission revealed a palpable right hemi-abdominal mass up to the level of the umbilicus, measuring roughly $8 \mathrm{~cm}$. in widest diameter, described as cystic, doughy to firm, non-tender, with limited mobility. Base serologic levels of human epididymis protein 4 (HE4), cancer antigen 125 (CA 125), and carbohydrate antigen 19-9 (CA 19-9) were determined, which showed elevated levels of HE4 and CA 125 at $502.2 \mathrm{pmol} / \mathrm{L}$ (normal range at $\leq 140 \mathrm{pmol} / \mathrm{L}$ ) and $689.3 \mathrm{U} / \mathrm{mL}$ (normal range at 0.00 - $35.00 \mathrm{U} / \mathrm{mL}$ ), as well as normal levels of CA 19-9 at $<1.20 \mathrm{U} / \mathrm{mL}$ (normal level at $0.00-37.00 \mathrm{U} / \mathrm{mL}$ ). Pelvic ultrasound showed a multiloculated solid cystic structure located posteroinferior to the uterus, described as having multiple papillations. A whole abdominal CT scan was also done, which showed a large thin-walled multiloculated heterogeneously enhancing, predominantly cystic mass in the abdomino-pelvic region with an irregular, heterogeneously enhancing soft tissue component located anterosuperiorly. Clinical diagnosis by the gynecologist and medical oncologist was of an ovarian new growth.

Total abdominal hysterectomy with bilateral salpingooophorectomy (TAHBSO) was done, as well as cytoreductive surgery and hyperthermic intraperitoneal chemotherapy with Cisplatin. Intraoperatively, the pelvoabdominal cystic mass was identified to be the right ovary. The right fallopian tube was stretched out and seen to envelop the right ovarian mass. There were multiple mesenteric implants, enlarged pelvic lymph nodes, and minimal perihepatic ascitic fluid.

The TAHBSO specimen was sent for histopathology. Gross examination revealed that the right ovary was converted into a yellow-tan to pink-tan, solid to cystic mass measuring $12.5 \times 11 \times 7.5 \mathrm{~cm}$. Sectioning of the mass revealed solid to cystic cut surfaces with egress of chocolate brown fluid with some clots. The solid portions had cream tan to yellow, soft to friable cut surfaces with areas of hemorrhage and necrosis.
Microscopic examination of the ovarian mass disclosed a malignant neoplasm with heterogeneous architecture, primarily composed of a carcinomatous and a sarcomatous component. Architecturally, there was blending of the two components in some areas. The carcinomatous component comprised roughly $60 \%$ of the tumor and was composed of infiltrative nests and papillae of epithelial tumor cells with enlarged, hyperchromatic, pleomorphic nuclei, and scant to moderate amount of cytoplasm (Figure 1). The sarcomatous component comprised roughly $30 \%$ of the tumor and was composed of sheets of atypical spindle cells with pleomorphic, hyperchromatic nuclei (Figure 2). Brisk mitotic activity is also noted. No heterologous mesenchymal elements were seen in the microsections obtained.

Another histological component was seen in the solid portion of the mass, comprising roughly $10 \%$ of the tumor. It had areas with microcystic and reticular architecture in a myxoid stroma (Figure 3). Present in this area are structures arranged in tubulo-papillary pattern with a central vascular core, lined by cuboidal to columnar epithelial-like cells (Figure 3, inset). With the heterogeneity of the tumor showing both glandular and mesenchymal components, an initial histopathologic diagnosis of carcinosarcoma in the right ovary was considered. However, due to the presence of areas with microcystic and reticular architecture not commonly seen in carcinosarcoma, the case was referred to a subspecialist. Immunohistochemical stains were also requested.

Immunohistochemical staining results showed positive staining for Paired-box gene $8($ Pax-8) and Estrogen Receptor (ERA) in the areas showing glandular differentiation (Figure 4). The same tumor population was negative for Sal-like protein 4 (SALL4) and Glypican-3. These results favor the presence of a malignant epithelial component. On the other hand, while some of the sarcomatous component showed diffuse positivity for CK,

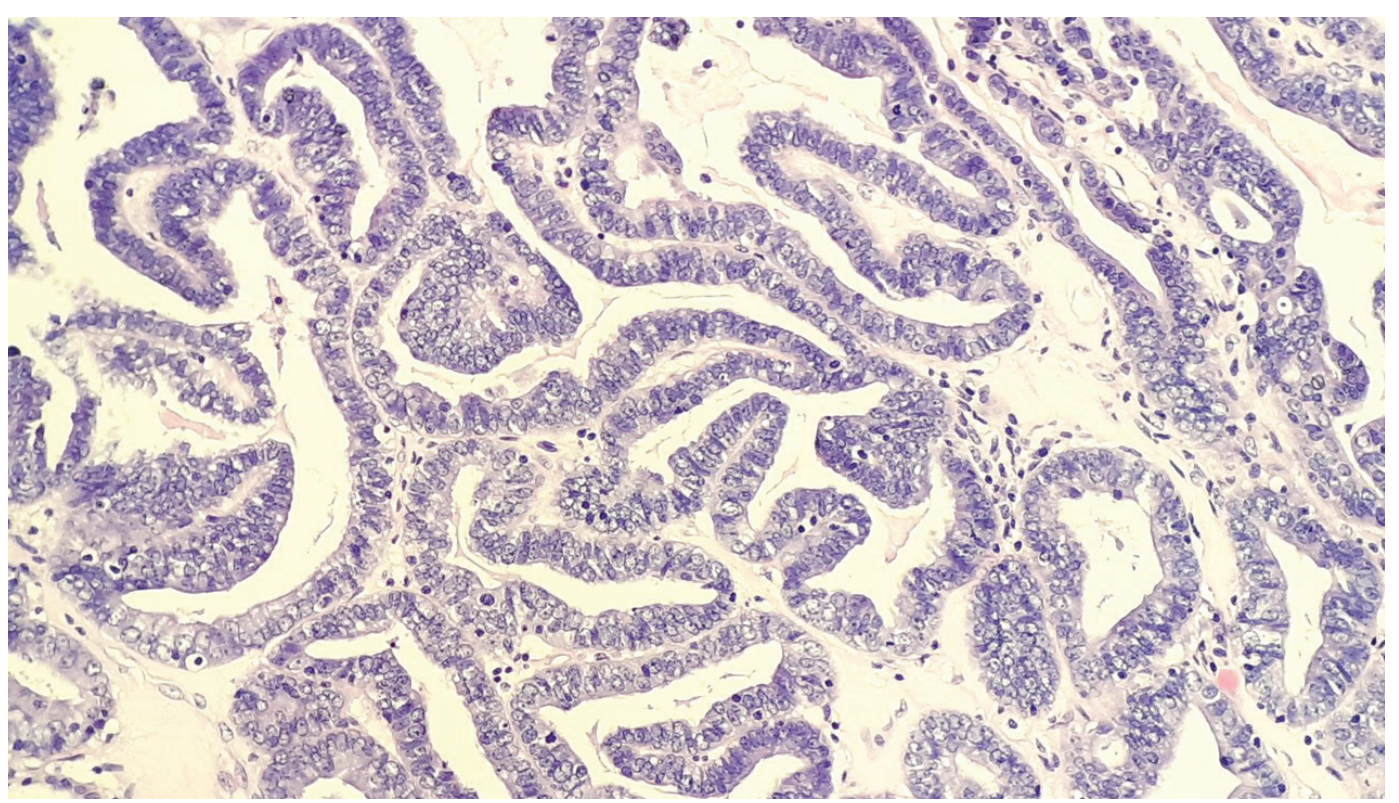

Figure 1. The carcinomatous component show glands and papillae lined with epithelial tumor cells with enlarged, hyperchromatic, pleomorphic nuclei and scant to moderate amount of cytoplasm (H\&E, 40x). 


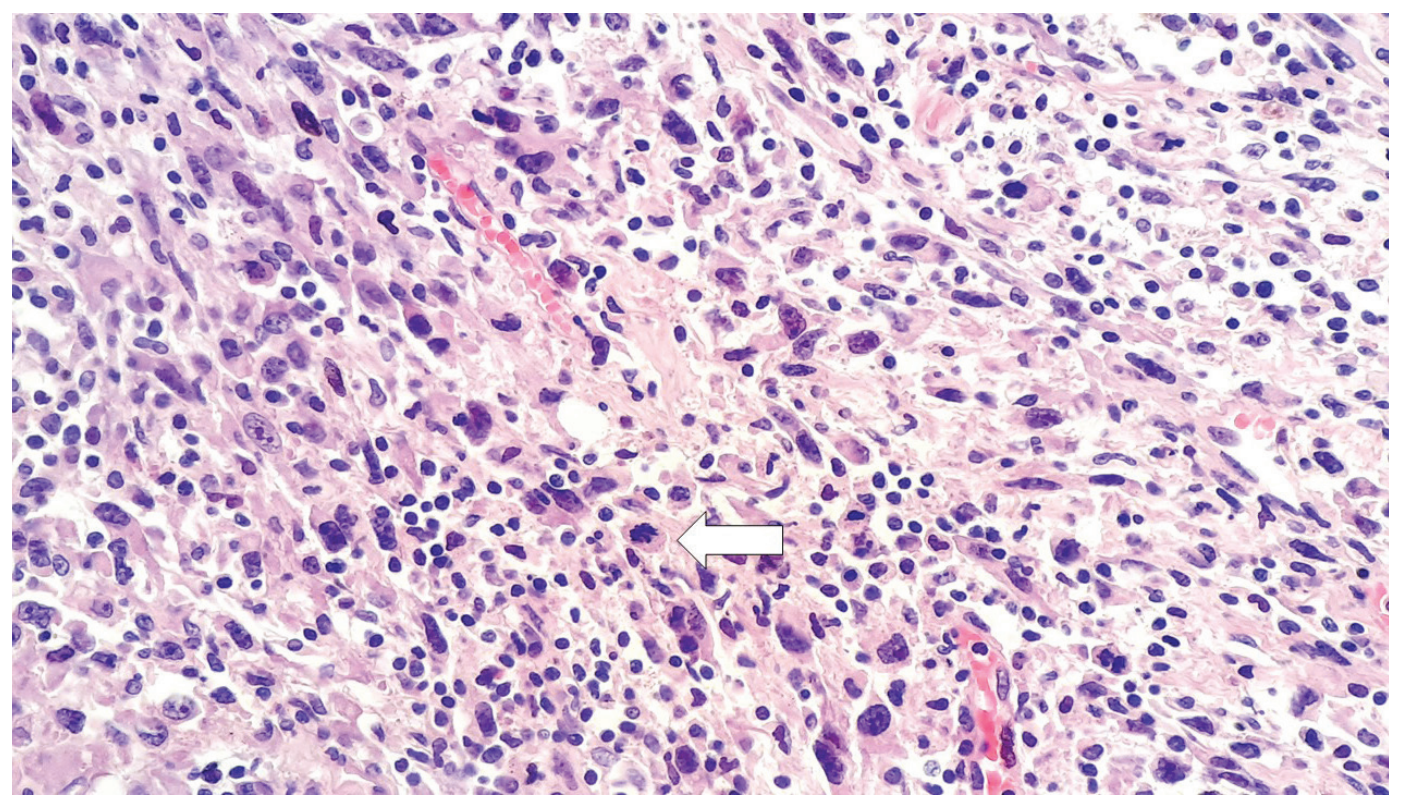

Figure 2. The sarcomatous component is composed of loose sheets of atypical spindle cells with pleomorphic, hyperchromatic nuclei. A mitotic figure can be seen (arrow) (H\&E, 40x).

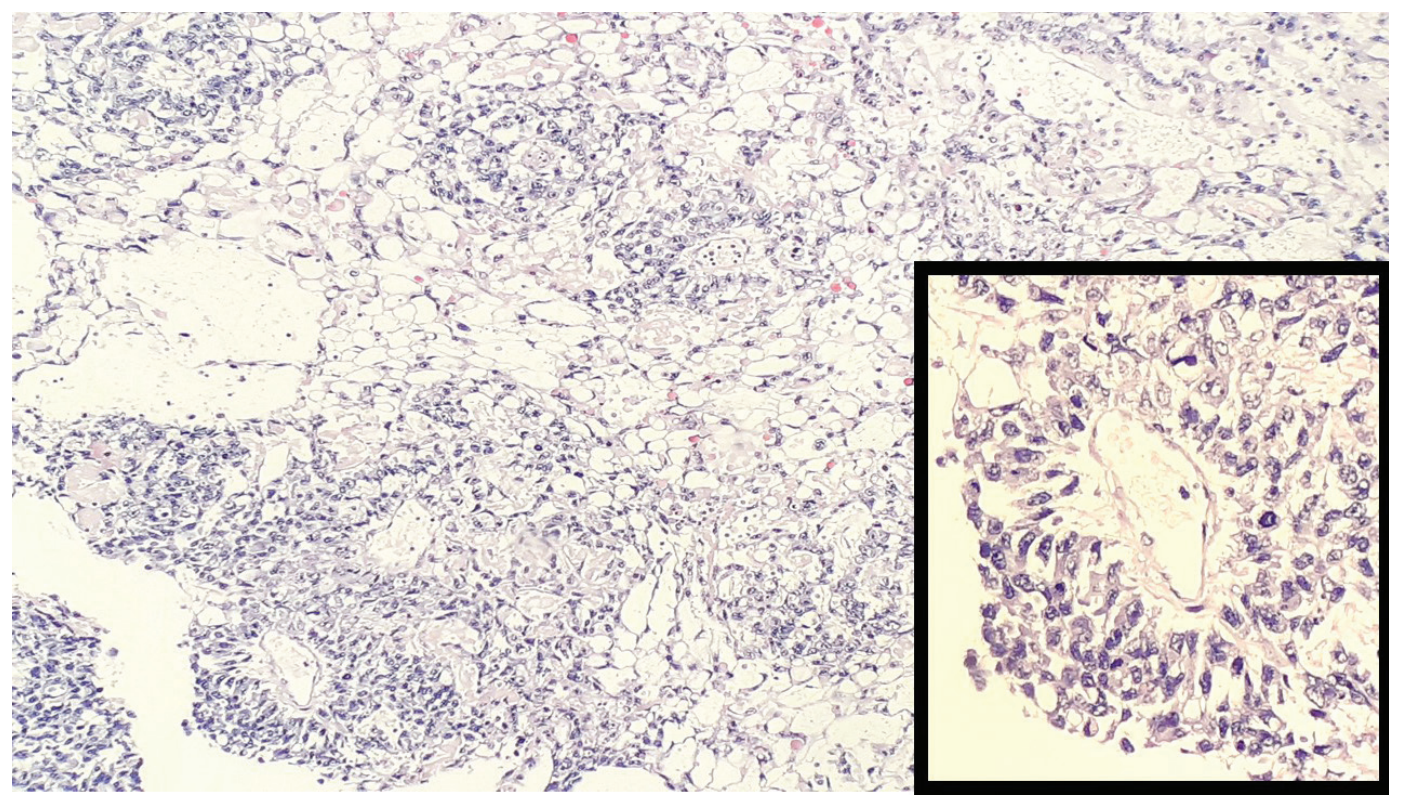

Figure 3. Yolk sac tumor component with microcystic and reticular architecture in a myxoid stroma. Inset. Schiller-Duval bodies with a central vascular core lined by cuboidal to columnar epithelial-like cells (H\&E, 10x, Inset 40x).

the higher grade areas showed diffuse positivity for CD10 and Vimentin, as well as negativity for CK, supporting its mesenchymal nature (Figure 5). An additional stain for PAX8 was done which was also negative. Desmin also stained negative, supporting the absence of heterologous rhabdomyoblasts. Lastly, SALL4 and Glypican-3 were both found to be expressed in the reticular and myxoid areas where the Schiller-Duval bodies were noted (Figure 6). The positivity of these stains supports a YST component. Alpha-fetoprotein (AFP) was also done, which was negative in this case.

Thus, a diagnosis of carcinosarcoma with a synchronous YST was made, which was seen to extend to the uterine serosa. The fallopian tubes and left ovary were uninvolved. Pelvic lymph node dissection revealed metastasis to the mesenteric lymph node. Liver nodules were also seen and a portion of the liver was submitted, but was ultimately negative for tumor. A mesenteric implant seen intraoperatively was positive for tumor $(2 \mathrm{~cm}$ in widest dimension). Peritoneal fluid was also sent for cytology which had atypical cells present; however, immunohistochemistry was not done to further elucidate the nature of these cells. The pathologic stage was determined as pT3bN1M0 based on AJCC (American Joint Committee on Cancer, $8^{\text {th }}$ edition), and FIGO (International Federation of Gynecology and Obstetrics) System Stage IIIB. 


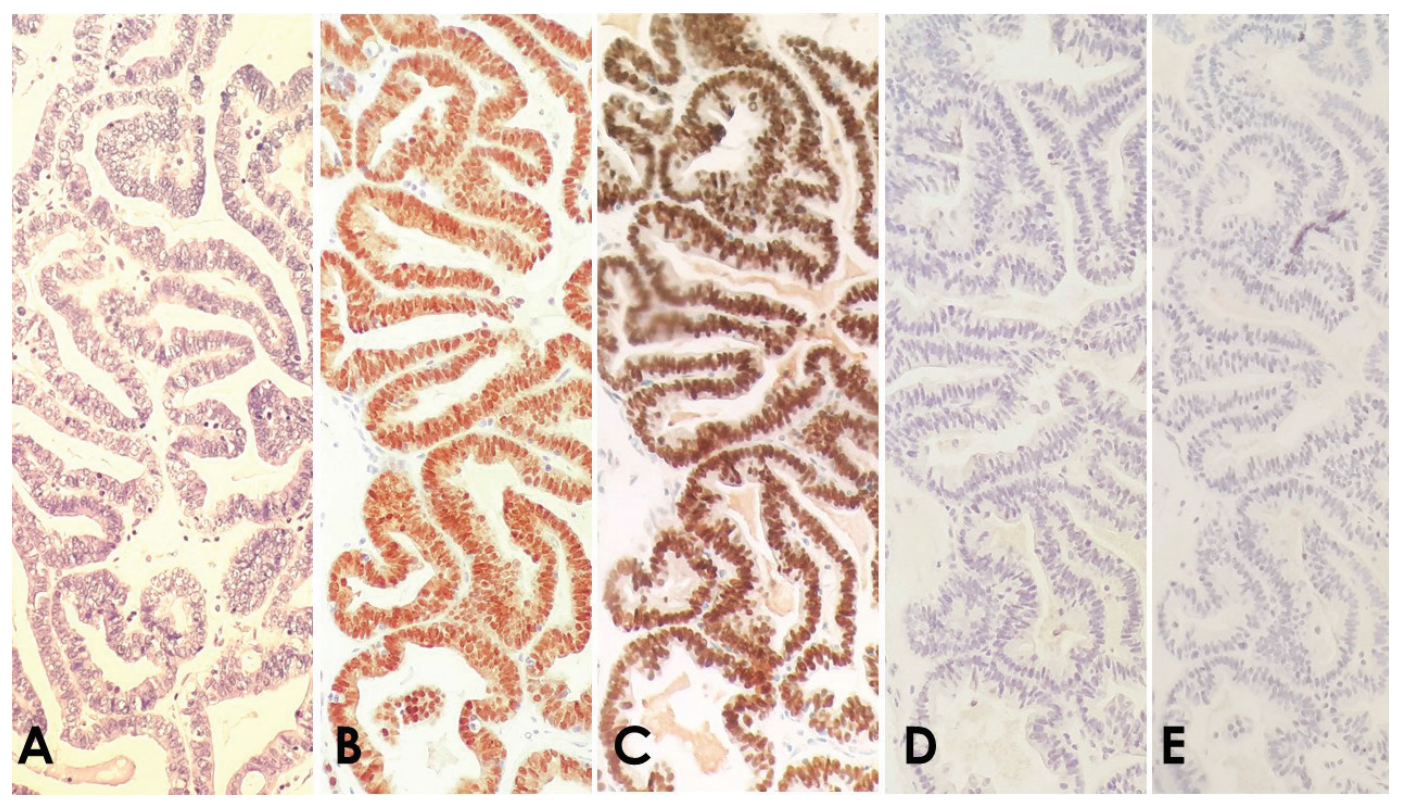

Figure 4. Carcinomatous component (A) H\&E, 10x; (B) PAX8 with diffuse positive nuclear staining, 10x; (C) ERA with diffuse positive nuclear staining, 10x; (D) SALL4 negative, 10x; (E) Glypican negative, 10x.

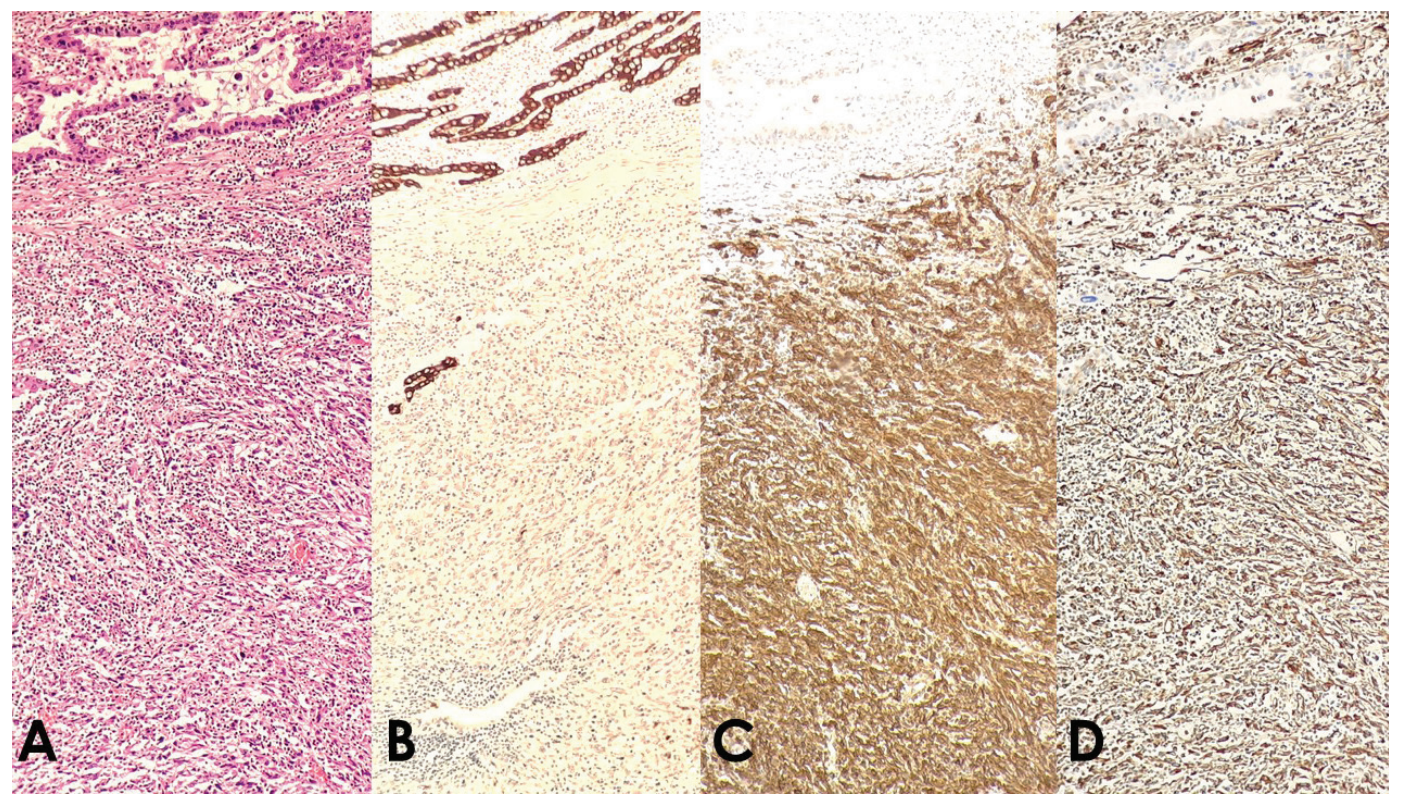

Figure 5. Sarcomatous component (A) H\&E stain, 10x; (B) CK negative, 10x; (C) CD10 with diffuse positive cytoplasmic staining, 10x; (D) Vimentin, with diffuse positive cytoplasmic staining, 10x.

Post-operatively, the patient received 10 cycles of chemotherapy with Paclitaxel-Carboplatin. Serology markers (carcinoembryonic antigen [CEA], HE4, and Ca-125) were monitored every 1-3 months (Figure 7). Serum AFP was determined roughly one month postoperatively and found to be within normal range at $<1.30 \mathrm{ng} / \mathrm{mL}$ (normal range at $0.00-8.00 \mathrm{ng} / \mathrm{mL}$ ). As of writing, the patient is currently alive and undergoing serologic monitoring. A repeat whole abdominal CT scan 5 months after the surgery revealed no discrete enhancing mass lesion or abnormal fluid collection in the surgical bed, but with a slight increase in the size of the enlarged precaval lymph node; enlarged lymph nodes are also seen in the para-aortic region.

\section{DISCUSSION}

YSTs occur rarely in elderly women, and when they do, they are mostly combined with other epithelial tumor components, ${ }^{6}$ unlike YSTs in younger women which present as pure or mixed with other germ cell components. As germ cells are not identified histologically in the ovaries of postmenopausal women, a direct origin of malignant neoplasms from germ cells is highly unlikely at that age, and the possibility of a collision tumor, in part derived from germ cells and in part from epithelium, is unlikely. Roth et al., proposed that in older women, the germ cell component arises through the transformation of the epithelial precursor neoplasm, referred to as "neometaplasia." 


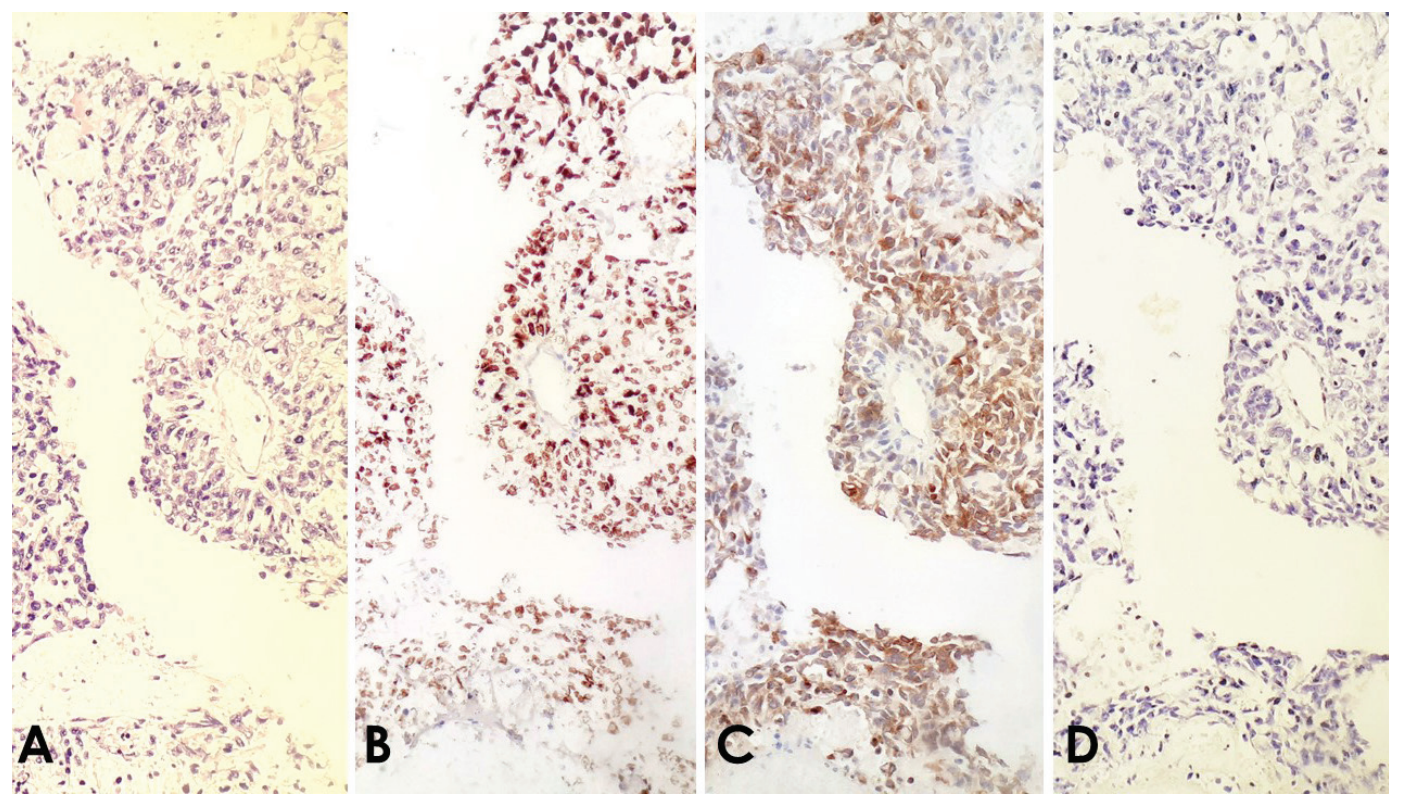

Figure 6. Yolk sac tumor component (A) H\&E, 10x; (B) SALL4 with diffuse positive nuclear staining, 10x; (C) Glycipan-3 with diffuse positive nuclear staining, 10x; (D) AFP negative, 10x.

McNamee et al., proposed the term "somaticallyderived YSTs" for such tumors which have undergone neometaplasia from epithelial precursors. $^{7}$ One point raised was how the YST components in their study were often diffusely positive with epithelial markers (EMA, BerEP4, CK7), which may reflect true epithelial differentiation in the YST component in keeping with the morphology of a glandular variant. In support of this shared origin between the different morphologic phenotypes, Ahn et al., were able to identify a tumor component with transitional histologic features between the YST, mucinous, and large-cell neuroendocrine tumor components in their specimen, so much so that the PAX8, glypican-3, and SALL4 immunoreactivity patterns were observed in these morphologically overlapping areas. ${ }^{8}$
The presence of spindle cells in the specimen infer the need to distinguish between a true carcinosarcoma from an endometrioid carcinoma with spindle cells, so called sarcomatoid carcinoma. The distinction between carcinosarcoma and sarcomatoid carcinomas lies on two things: first, the degree of atypia of the glandular and sarcomatous components, and second, whether or not the two components are spatially distinct from one another. ${ }^{9}$ In our case, both epithelial and sarcomatous components were notably high grade with increased cellular pleomorphism and mitotic activity. In addition, although some areas in our case showed intermingling of the epithelial and sarcomatous components, carcinosarcomas in the ovary are noted to show two distinct components but are typically intermingled with one another. Diffuse

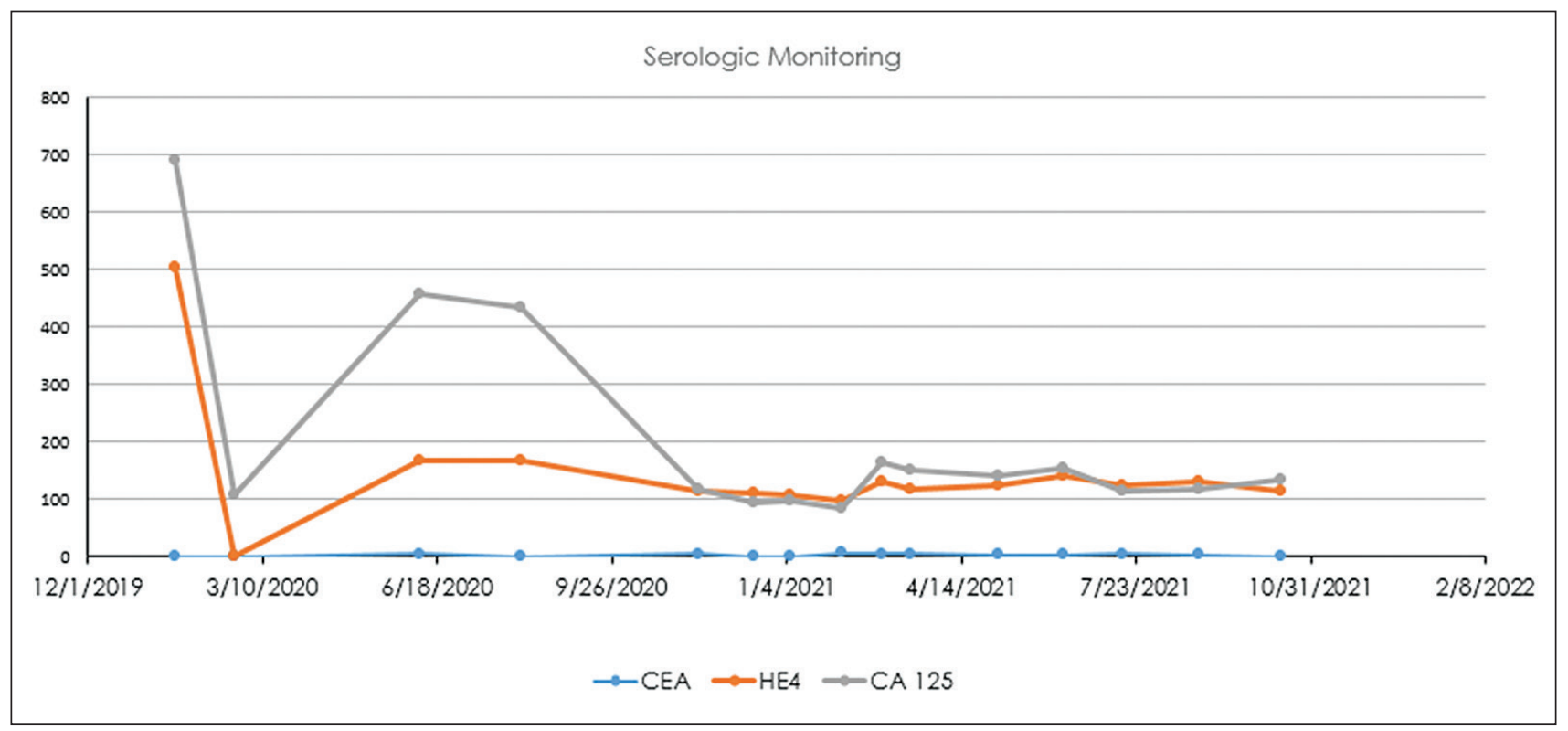

Figure 7. Serologic Markers. CEA, HE4, and CA-125 levels were monitored post-operatively, which showed a decreasing trend. 
positivity with CD10 and vimentin exclusively in the spindle cell areas, as well as negativity for PAX8 and CK in the high-grade spindle cell areas, is consistent with the sarcomatous component of a carcinosarcoma.

The most common and distinctive patterns for YST in postmenopausal women include the reticular, or microcystic, and the endodermal, or pseudopapillary pattern. ${ }^{10}$ Schiller-Duval bodies are most often associated with the latter and are diagnostic of YSTs. These were seen in our case. On the other hand, endometrioid carcinoma is the most common epithelial component in mixed YSTs in post-menopausal women ${ }^{6,10}$ Endometriosis often coexists with these tumors as a precursor lesion. ${ }^{10,11}$ Only five cases had carcinosarcoma as the epithelial component since its first mention in English literature. ${ }^{3-5}$

PAX8 was used to identify the epithelial component in our case. PAX8, a member of the mammalian paired box genes 1 to 9 that encodes a transcription factor involved in embryogenesis, is thought to play a regulatory role in cell fate decisions during the development of Müllerian organs. ${ }^{12}$ As it is associated with Müllerian development, it is negative in those ovarian tumors not associated with Müllerian surface epithelium, including GCTs and sex cord stromal tumors. Other immunohistochemical stains that can be used for differentiating ovarian YST from endometrioid carcinoma include Epithelial membrane antigen (EMA), CK7, and BerEP4.7.13 These stains are generally negative or only focally positive for YST, and are generally diffusely positive for Müllerian adenocarcinoma.

AFP is a stain that has conventionally been positive in YST. However, YSTs often express AFP only focally, as demonstrated in the study by Roma et al., so much so that positivity for AFP did not reliably establish the diagnosis of YST in their case. ${ }^{12}$ Newer markers glypican-3 and SALL4 are useful in the identification of the YST component. ${ }^{6}$ Positive cytoplasmic staining for glypican-3, an oncofetal protein expressed in fetal liver and malignant tumors of hepatocytic lineage, is more sensitive than AFP but not as specific. ${ }^{11}$ On the other hand, although SALL4, a zinc finger transcription factor, is not specific for YSTs amongst germ cell neoplasms, it is a specific and sensitive marker for GCTs and its positive nuclear staining is useful in distinguishing YSTs from non-GCTs such as carcinomas. ${ }^{7}$ In our case, the YST component was positive for glypican 3 and SALL4, and negative for AFP.

As the YST component arises by transformation of an epithelial ovarian neoplasm resulting in a different molecular pathway than those of GCTs in younger patients, it may be less sensitive to chemotherapy than those that arise de novo. ${ }^{14,15}$ Because of its rarity, there are currently no systemic treatment guidelines available. As with pure GCTs, Bleomycin-Cisplatin-Etoposide (BEP) chemotherapy is a potential choice for treatment and may be effective not only in the germ cell but also in the epithelial component. ${ }^{10}$ On the other hand, as what is recommended for carcinosarcomas, ${ }^{14}$ adjuvant therapy with cisplatin or carboplatin-based chemotherapy, such as carboplatin and paclitaxel, has also been found to be effective. ${ }^{6,13,16}$ The core of effectivity in both treatments rests on the platinum ingredient, which was found to be effective against both epithelial ovarian cancer and GCTs. ${ }^{10}$ For our patient, she has undergone Cytoreductive Surgery with Hyperthermic Intraperitoneal Chemotherapy (Cisplatin). Post-operatively she has also received 10 cycles of chemotherapy with Paclitaxel-Carboplatin.

The biologic behavior and prognostic significance of a mixed YST and carcinosarcoma in the ovary of postmenopausal women are unclear due to the limited case numbers and insufficient clinical information. In general, YST in the postmenopausal population is associated with a distinct biologic behavior characterized by poor prognosis even with early-stage disease, whether or not an epithelial component is detected. ${ }^{13}$ Recurrence occurs within 7 months despite systemic chemotherapy, ${ }^{6}$ and mortality within 8 months to 2 years. ${ }^{6,10,15}$ Some studies have suggested that the disease-free survival of patients with mixed YST-epithelial carcinoma follows the epithelial component. The median survival for such cases is estimated to be less than 18 months to less than 2 years. ${ }^{16}$ As of writing, our patient is currently alive roughly 20 months after being diagnosed with the disease at FIGO Stage IIIB.

Elevated tumor markers have been shown to be an independent poor prognostic indicator. ${ }^{10}$ Serum markers may normalize during chemotherapy, but this may reflect regression of only one component of the mixed lesion. Our case did not have available serum levels of AFP before surgery as YST in postmenopausal patients is very rare and its diagnosis was not suspected before surgery. Levels of CA-125 were elevated which may be attributed to the epithelial component in our case.

\section{CONCLUSION}

We report a case of mixed YST with carcinosarcoma in a postmenopausal female. Because of its rarity, the combination of histomorphology and immunohistochemical stains may aid in its identification. Though YST components may be less responsive to traditional germ-cell tumor chemotherapy because of its differentiation from epithelial components, adjuvant Platinum-based chemotherapy is useful as it targets both epithelial ovarian tumors and germ-cell tumors. Ovarian YSTs in postmenopausal women have a poor prognosis, and therefore more active treatment and post-operative monitoring are needed.

\section{ACKNOWLEDGMENTS}

The authors acknowledge Dr. Jose Moran and Dr. Charles Joseph Bernardo for their contribution to this report.

\section{ETHICAL CONSIDERATION}

Patient consent was obtained before submission of the manuscript.

\section{STATEMENT OF AUTHORSHIP}

All authors certified fulfillment of ICMJE authorship criteria. 


\section{AUTHOR DISCLOSURE}

The authors declared no conflict of interest.

\section{FUNDING SOURCE}

None.

\section{REFERENCES}

1. Shaaban AM, Rezvani M, Elsayes KM, et al. Ovarian malignant germ cell tumors: cellular classification and clinical and imaging features. Radiographics. 2014;34(3):777-801. PMID: 24819795. https://doi.org/ 10.1148/rg.343130067.

2. Rutgers JL, Young RH, Scully RE. Ovarian yolk sac tumor arising from an endometrioid carcinoma. Hum Pathol. 1987;18(12):1296-9. PMID: 3679203. https:// doi.org/10.1016/s0046-8177(87)80418-5.

3. Nogales F, Bergeron C, Carvia R, Alvaro T, Fulwood H. Ovarian endometrioid tumors with yolk sac tumor component, an unusual form of ovarian neoplasm. Analysis of six cases. Am J Surg Pathol.1996;20(9):1056-66. PMID: 8764742. https:// doi.org/10.1097/00000478-199609000-00003.

4. Garcia-Galvis OF, Cabrera-Ozoria C, Fernandez JA, et al. Malignant Müllerian mixed tumor of the ovary associated with yolk sac tumor, neuroepithelial and trophoblastic differentiation (teratoid carcinosarcoma). Int J Gynecol Pathol 2008; 27: 515-20. PMID: 18753971. https://doi.org/10.1097/ PGP.0b013e31817b06c7.

5. Fadare O, Shaker N, Alghamdi A, et al. Endometrial tumors with yolk sac tumor-like morphologic patterns or immunophenotypes: an expanded appraisal. Mod Pathol. 2019;32(12):1847-60. PMID: 31375771. https://doi.org/10.1038/s41379-019-0341-6.

6. Roth LM, Talerman A, Levy T, Sukmanov O, Czernobilsky B. Ovarian yolk sac tumors in older women arising from epithelial ovarian tumors or with no detectable epithelial component. Int J Gynecol Pathol. 2011;30(5):442-51. PMID: 21804392. https://doi.org/10.1097/PGP.0b013e3182164386.

7. McNamee T, Damato S, McCluggage WG. Yolk sac tumours of the female genital tract in older adults derive commonly from somatic epithelial neoplasms: somatically derived yolk sac tumours. Histopathol. 2016;69(5):739-51. PMID: 27334714. https://doi.org/ 10.1111/his.13021.
8. Ahn H, Oh MH, Cho HD, et al. Ovarian yolk sac tumor with epithelial tumor component in a postmenopausal woman - case report and literature review. Int J Clin Exp Pathol. 2020;13(9):2401-6. PMID: 33042351. PMCID: PMC7539866.

9. Rabban JT. Distinction of low-grade endometrioid carcinoma in endometrial samplings from high-grade mimics: a practical pattern-based approach. Diagn Histopathol. 2020;26(6):237-47.

10. Boussios S, Attygalle A, Hazell S, et al. Malignant ovarian germ cell tumors in postmenopausal patients: The Royal Marsden experience and literature review. Anticancer Res. 2015;35(12):6713-22. PMID: 26637887.

11. Nogales FF, Preda O, Nicolae A. Yolk sac tumours revisited. A review of their many faces and names. Histopathology. 2012;60(7):1023-33. PMID: 22008025. https://doi.org/10.1111/j.1365-2559.2011.03889.

12. Roma AA, Przybycin CG. Yolk sac tumor in postmenopausal patients: pure or associated with adenocarcinoma, a rare phenomenon. Int J Gynecol Pathol. 2014;33(5):477-82. PMID: 25083963. https:// doi.org/10.1097/PGP.0000000000000078.

13. Taranto P, Carvalho FM, Roithmann S, Maluf FC. Ovarian yolk sac tumor coexisting with epithelial ovarian cancer: an aggressive rare entity. Gynecol Oncol Rep. 2017;22:37-9. PMID: 29204510. PMCID: PMC5689461. https://doi.org/10.1016/j.gore.2017.09. 004.

14. Lopez JM, Malpica A, Deavers MT, Ovarian yolk sac tumor associated with endometrioid carcinoma and mucinous cystadenoma of the ovary. Ann Diagn Pathol. 2003;7(5):300-5. PMID: 14571433. https://doi. org/10.1016/s1092-9134(03)00081-9.

15. Wang Y, Yang J, Yu M, et al. Ovarian yolk sac tumor in postmenopausal females: a case series and literature review. Medicine (Baltimore). 2018;97(33):e11838. PMID: 30113473. PMCID: PMC6112915. https://doi. org/10.1097/MD.0000000000011838.

16. Del Carmen MG, Birrer M, Schorge JO. Carcinosarcoma of the ovary: a review of the literature. Gynecol Oncol. 2012;125(1):271-7. PMID: 22155675. https://doi.org/10.1016/j.ygyno.2011.12.418.

\footnotetext{
Disclaimer: This journal is OPEN ACCESS, providing immediate access to its content on the principle that making research freely available to the public supports a greater global exchange of knowledge. As a requirement for submission to the PJP, all authors have accomplished an AUTHOR FORM, which declares that the ICMJE criteria for authorship have been met by each author listed, that the article represents original material, has not been published, accepted for publication in other journals, or concurrently submitted to other journals, and that all funding and conflicts of interest have been declared. Consent forms have been secured for the publication of information about patients or cases; otherwise, authors have declared that all means have been exhausted for securing consent.
} 